\title{
Parabronchial smooth muscle constitutes an airway epithelial stem cell niche in the mouse lung after injury
}

\author{
Thomas Volckaert, ${ }^{1,2,3}$ Erik Dill, ${ }^{1}$ Alice Campbell,, ${ }^{1}$ Caterina Tiozzo, ${ }^{4}$ Susan Majka, ${ }^{5}$ \\ Saverio Bellusci, 4,6 and Stijn P. De Langhe ${ }^{1}$
}

\begin{abstract}
1Department of Pediatrics, Division of Cell Biology, National Jewish Health, Denver, Colorado, USA. ${ }^{2}$ Department for Molecular Biomedical Research, Unit of Molecular Signal Transduction in Inflammation, VIB, Ghent, Belgium. 3Department of Biomedical Molecular Biology, Ghent University, Ghent, Belgium. ${ }^{4}$ Developmental Biology Program, Division of Surgery, Saban Research Institute of Children's Hospital Los Angeles, Los Angeles, California, USA. ${ }^{5}$ Department of Medicine, University of Colorado Denver, Aurora, Colorado, USA. ${ }^{6}$ Excellence Cluster in Cardio-Pulmonary Systems, University of Giessen Lung Center, Giessen, Germany.
\end{abstract}

\begin{abstract}
During lung development, parabronchial SMC (PSMC) progenitors in the distal mesenchyme secrete fibroblast growth factor 10 (Fgf10), which acts on distal epithelial progenitors to promote their proliferation. $\beta$-catenin signaling within PSMC progenitors is essential for their maintenance, proliferation, and expression of Fgf10. Here, we report that this Wnt/Fgf10 embryonic signaling cascade is reactivated in mature PSMCs after naphthalene-induced injury to airway epithelium. Furthermore, we found that this paracrine Fgf10 action was essential for activating surviving variant Clara cells (the cells in the airway epithelium from which replacement epithelial cells originate) located at the bronchoalveolar duct junctions and adjacent to neuroendocrine bodies. After naphthalene injury, PSMCs secreted Fgf10 to activate Notch signaling and induce Snail expression in surviving variant Clara cells, which subsequently underwent a transient epithelial to mesenchymal transition to initiate the repair process. Epithelial Snai1 expression was important for regeneration after injury. We have therefore identified PSMCs as a stem cell niche for the variant Clara cells in the lung and established that paracrine Fgf10 signaling from the niche is critical for epithelial repair after naphthalene injury. These findings also have implications for understanding the misregulation of lung repair in asthma and cancer.
\end{abstract}

\section{Introduction}

Throughout life, multicellular organisms must generate new cells to maintain the integrity of their tissues, but the capacity to repair tissue damage may fail after repeated injury and with age. The adult lung is a vital and complex organ that normally turns over very slowly and is one of the few organs that has a direct interface with the outside environment. The epithelial cells that line the airways are constantly exposed to potential toxic agents and pathogens and therefore must be able to respond quickly and effectively to cellular damage. The cellular hallmark of lung repair after naphthalene injury is a rapid proliferative response ultimately leading to restoration of the airway epithelium and function. The origin of the cells that replace the injured airway epithelium have been shown to be naphthalene-resistant variant Clara cells located at the bronchoalveolar duct junctions (BADJs) (1) and neuroendocrine bodies (NEBs) (2). However, little is known about the activation mechanism of these latent stem cells (reviewed in refs. 3, 4). Knowledge about the pathways involved in the activation of these latent epithelial stem cells could enable new therapeutic strategies for treatment of lung disease.

During lung development, fibroblast growth factor 10 (Fgf10) acts on the distally located epithelial progenitors to prevent their differentiation and promote their proliferation (5-8). Fgf10 is secreted by parabronchial SMC (PSMC) progenitors in the distal mesenchyme, and its expression is dependent on $\beta$-catenin signaling

Authorship note: Thomas Volckaert and Erik Dill contributed equally to this work. Conflict of interest: The authors have declared that no conflict of interest exists. Citation for this article: J Clin Invest. 2011;121(11):4409-4419. doi:10.1172/JCI58097.
(9-11). Here we report that this Wht/Fgf10 embryonic signaling cascade is reactivated in mature PSMCs after naphthalene-induced Clara cell epithelial injury. Using lineage tracing and loss- and gainof-function studies of the Fgf, Wnt, and Notch pathways, we demonstrated that this paracrine Fgf10 action was essential for activation of the surviving variant Clara cells located at the BADJs and adjacent to the NEBs. After naphthalene injury, PSMCs secreted Fgf10 to activate Notch signaling and induce Snail expression in surviving variant Clara cells, which subsequently underwent a transient epithelial to mesenchymal transition (EMT) to initiate the repair process. We also demonstrated that Snai1 expression in Clara cells undergoing repair was important for the proper restoration and function of the airway epithelium. We propose that PSMCs constitute a stem cell niche for the variant Clara cells in the lung and that paracrine Fgf10 signaling from the niche is critical for epithelial repair after naphthalene injury.

\section{Results}

Reactivation of Wnt signaling reinduces Fgf 10 expression in PSMCs 3 days after naphthalene-mediated Clara cell injury. We previously showed that a large proportion of PSMCs in the lung are derived from Fof10-expressing cells in the distal mesenchyme during early lung development (10) and that treatment of embryonic lung explants with Dickkopf1 (Dkk1), a canonical Wnt/ $\beta$-catenin inhibitor, impairs PSMC differentiation (12). In addition, we have shown that amplification of these PSMC progenitors, as well as their Fof10 expression, is regulated by mesenchymal $\beta$-catenin signaling and that deletion of $\beta$-catenin leads to their premature differentiation into PSMCs (9). During lung development, Fgf10 signaling is 

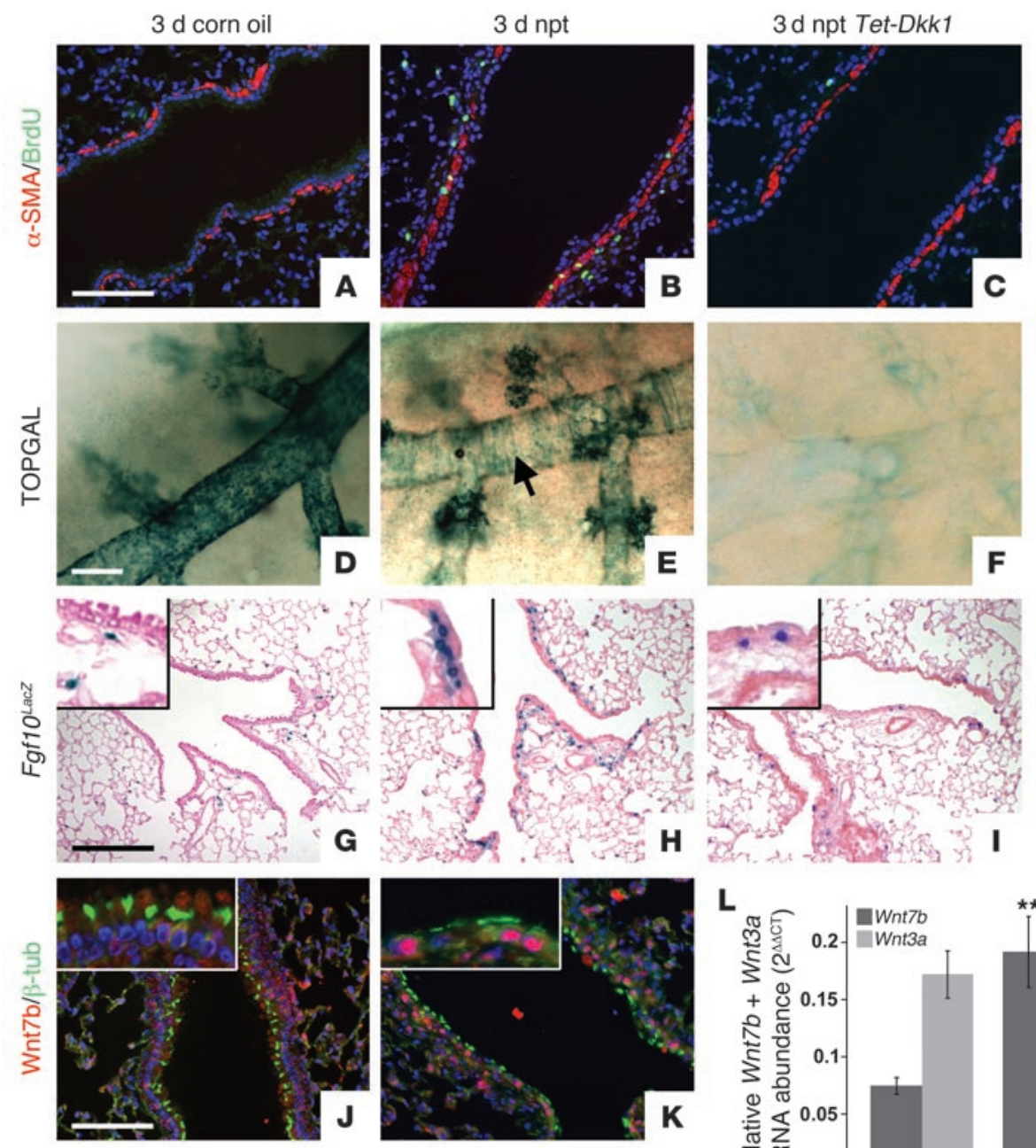

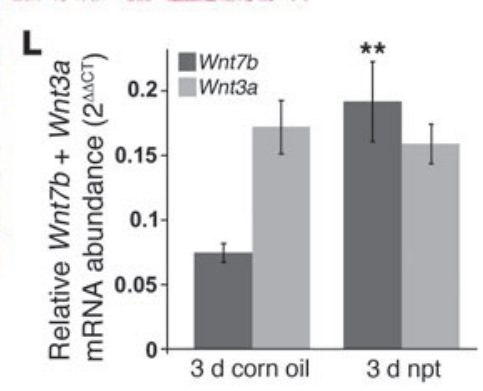

Figure 1

Wnt7b expressed by surviving ciliated cells induces Fgf10 expression in PSMCs 3 days after naphthalene-mediated Clara cell injury. (A-C) Immunostaining for proliferation marker BrdU and SMC marker $\alpha$-SMA on 2-month-old WT lungs 3 days after corn oil treatment (A), WT lungs 3 days after naphthalene (npt) treatment (B), and Rosa26-rtTa;Tet-Dkk1 lungs 3 days after naphthalene treatment (C). (D-F) $\beta$-gal staining on 2-month-old TOPGAL lungs 3 days after corn oil treatment (D), TOPGAL lungs 3 days after naphthalene treatment (E), and Rosa26-rtTa;Tet-Dkk1;TOPGAL lungs 3 days after naphthalene treatment (F). Arrow in E denotes TOPGAL activation in PSMCs. (G-I) $\beta$-gal staining on 2 -month-old Fgf10 LacZ lungs 3 days after corn oil treatment (G), Fgf10 LacZ lungs 3 days after naphthalene treatment (H), and Rosa26-rtTa;Tet-Dkk1;Fgf10LacZ lungs 3 days after naphthalene treatment (I). Insets are enlarged $\times 4$. Note that we identified the blue cells in alveolar compartment as lipofibroblasts (S.P. De Langhe, unpublished observations). ( $\mathbf{J}$ and $\mathbf{K}$ ) Immunostaining for ciliated cell marker $\beta$-tubulin and Wnt7b on 2-month-old WT lungs 3 days after corn oil treatment $(\mathbf{J})$ or naphthalene treatment $(\mathbf{K})$. Insets are enlarged $\times 3$. (L) qPCR analysis of relative Wnt7b and Wnt3a mRNA abundance in 2-month-old WT lungs 3 days after treatment with corn oil versus naphthalene. ${ }^{* \star} P<0.01$ vs. respective control. $n=3$. Scale bars: $100 \mu \mathrm{m}(\mathbf{A}-\mathbf{C}, \mathbf{J}$, and K); $250 \mu \mathrm{m}(\mathbf{D}-\mathbf{F}) ; 200 \mu \mathrm{m}(\mathbf{G}-\mathbf{I})$.

critical in the maintenance of lung epithelial progenitors $(5,8,13)$, in part through direct activation of epithelial $\beta$-catenin signaling. Although the Fgf/ $\beta$-catenin signaling axis in lung development is relatively well described, it is unclear whether this signaling pathway is recapitulated in adult lungs after lung epithelial injury as part of the repair process.

BrdU labeling of lungs 3 days after naphthalene-mediated epithelial injury indicated a robust proliferation of the PSMCs starting 3 days before injury prevented the induction of TOPGAL activity in PSMCs as well as in variant Clara cells 3 days after naphthalene injury (Figure $1 \mathrm{~F}$ ). We then used $\mathrm{Fg} f 10^{\mathrm{LacZ}}$ reporter mice $(10,15)$, which express $L a c Z$ under control of the Fgf10 promoter, to analyze Fgf10 expression in 2-month-old adult lungs during normal homeostasis, 3 days after naphthalene injury, and 3 days after naphthalene injury while overexpressing Dkk1. PSMCs, unlike their progenitors during development, did not express Fgf10 

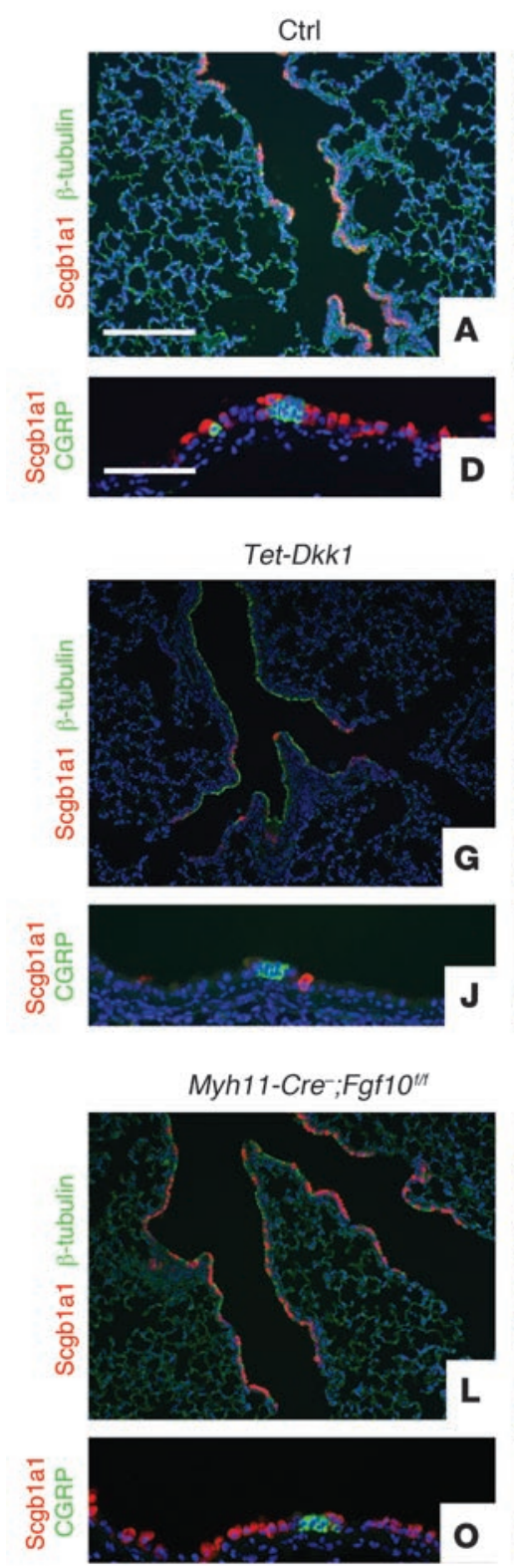
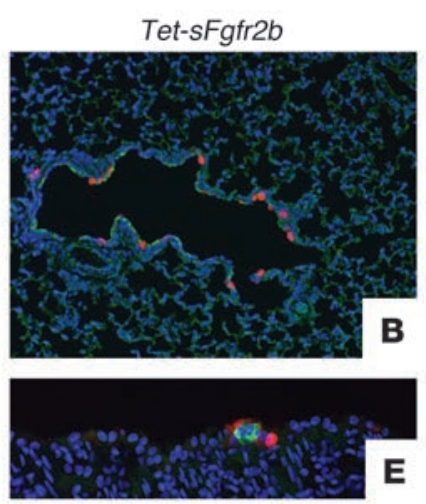

Tet-Dkk1 + Tet-Fgf10
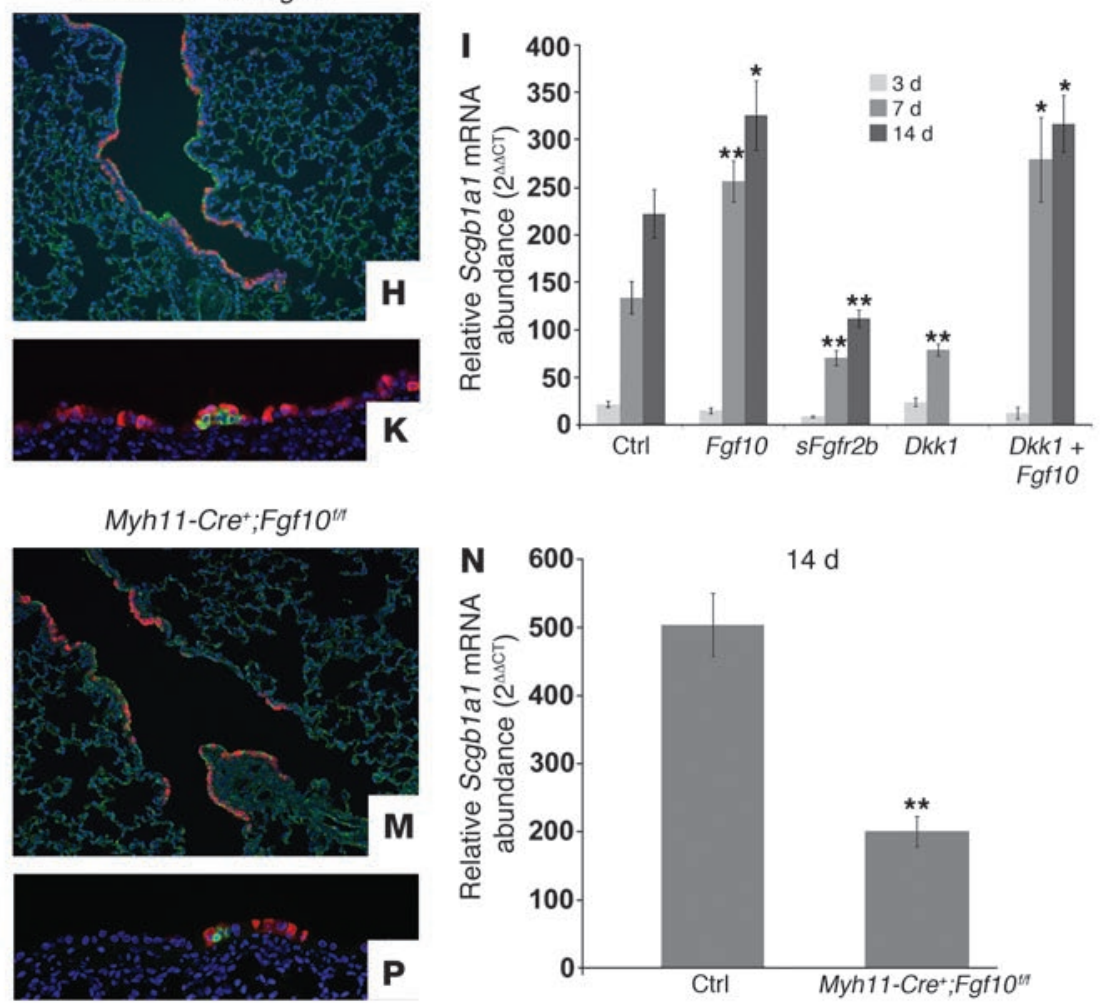

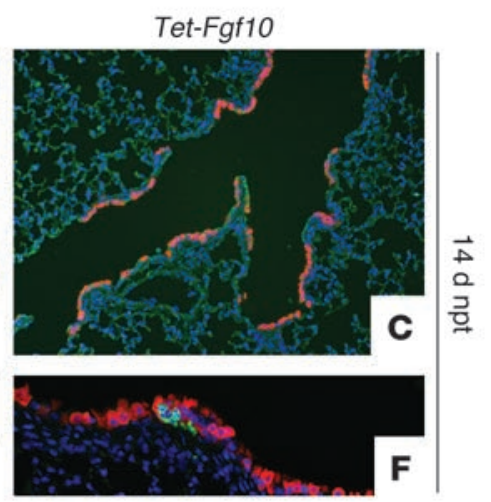

$\overrightarrow{1}$
$\frac{2}{2}$
$\frac{0}{7}$

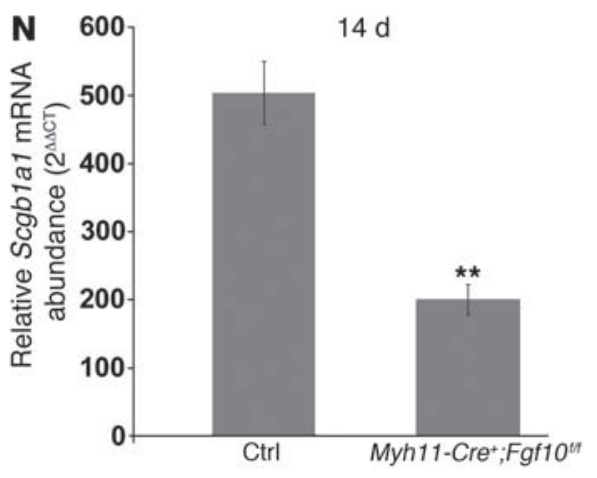

\section{Figure 2}

Wnt-induced Fgf10 secreted by PSMCs is essential for epithelial repair after naphthalene injury. (A-C, G, H, L, and M) Immunostaining for Clara cell marker Scgb1a1 and ciliated cell marker $\beta$-tubulin on lungs 14 days after naphthalene treatment isolated from control (A), dox-induced Rosa26-rtTa;Tet-sFgfr2b (B), dox-induced Rosa26-rtTA;Tet-Fgf10 (C), dox-induced Rosa26-rtTa;Tet-Dkk1 (G), dox-induced Rosa26-rtTa; Tet-Dkk1;Tet-Fgf10 (H), Myh11-Cre-;Fgf10 fl/fl (L), and Myh11-Cre+;Fgf10 ${ }^{f / f l}(\mathbf{M})$ mice. (D-F, J, K, O, and P) Immunostaining for Scgb1a1 and neuroendocrine marker CGRP on lungs 14 days after naphthalene treatment isolated from control (D), dox-induced Rosa26-rtTa;Tet-sFgfr2b (E), dox-induced Rosa26-rtTA;Tet-Fgf10 (F), dox-induced Rosa26-rtTa;Tet-Dkk1 (J), dox-induced Rosa26-rtTa;Tet-Dkk1;Tet-Fgf10 (K), Myh11-Cre-; $\mathrm{Fgf1O}^{\mathrm{fl/fl}}(\mathbf{O})$, and Myh11-Cre+;Fgf10 $\mathrm{O}_{\text {fl/fl }}$ (P) mice. (I) qPCR analysis of relative Scgb1a1 mRNA abundance of adult lungs from control, Rosa26-rtTa; Tet-sFgfr2b, Rosa26-rtTA-Tet-Fgf10, Rosa26rtTa;Tet-Dkk1, and Rosa26-rtTa;Tet-Dkk1;Tet-Fgf10 mice 3, 7, and 14 days after naphthalene

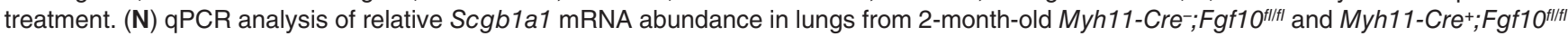
mice 14 days after naphthalene treatment. ${ }^{* *} P<0.01$, ${ }^{*} P<0.05$ vs. respective control. $n \geq 3$. Scale bars: $200 \mu \mathrm{m}(\mathbf{A}-\mathbf{C}, \mathbf{G}, \mathbf{H}, \mathbf{L}$, and $\mathbf{M}) ; 100 \mu \mathrm{m}$ (D-F, J, K, O, and P).

during normal homeostasis (Figure $1 \mathrm{G}$ and Supplemental Figure 1, A and E), but showed strong induction of Fgf10 expression 3 and 7 days after injury (Figure $1 \mathrm{H}$ and Supplemental Figure 1, $\mathrm{B}$ and $\mathrm{F}$ ) that was inhibited by Dkk1 overexpression (Figure 1I and Supplemental Figure 1, C and N). Note that we have identified the
Fgf10-expressing cells in the alveolar compartment (Figure 1, G-I) as lipofibroblasts (S.P. De Langhe, unpublished observations).

Dkk1 acts by inducing degradation of the LRP5/ 6 coreceptor, thereby preventing the binding of a Wnt ligand to the Frizzled receptor $(16,17)$. We therefore compared the expression levels of 

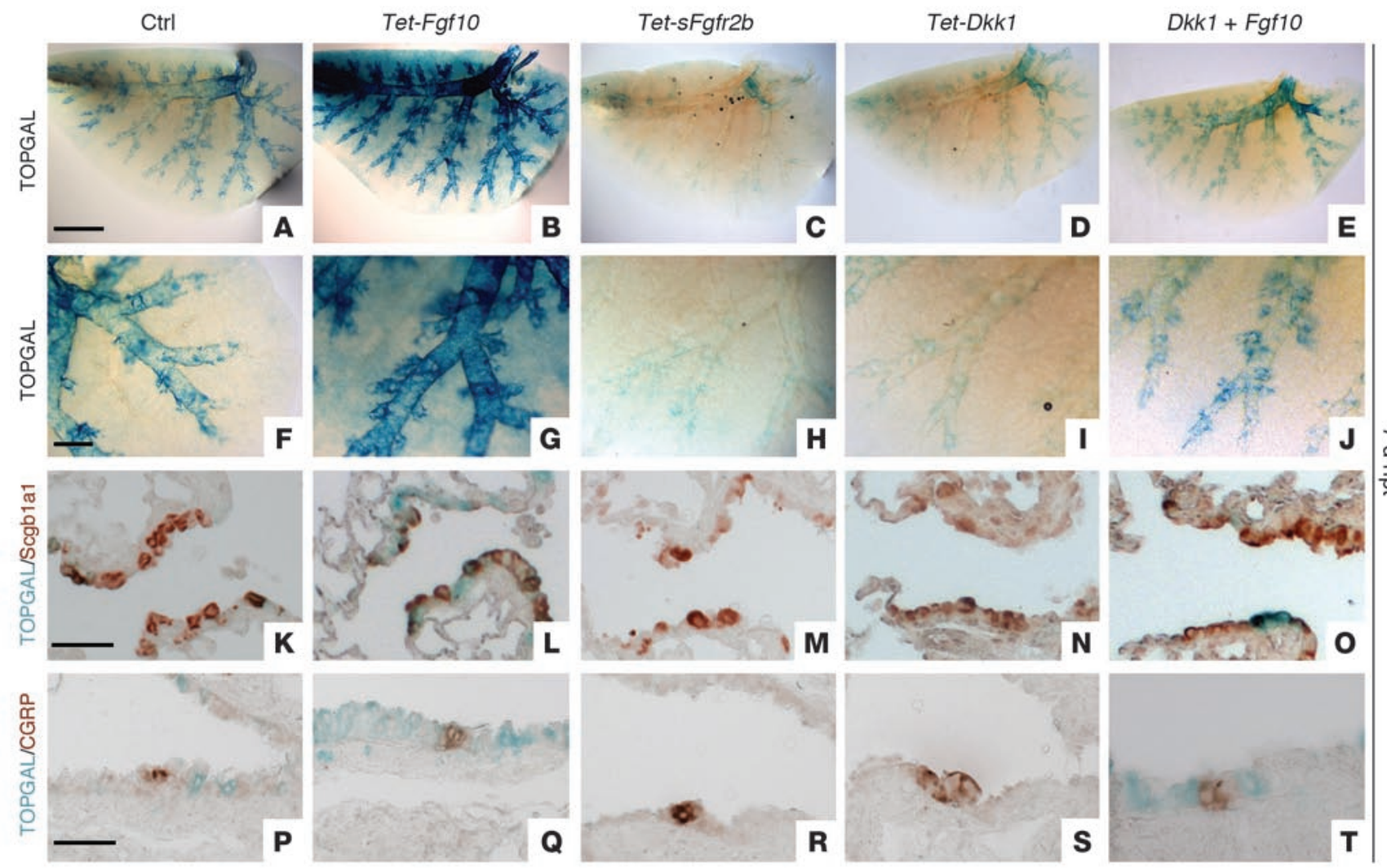

\section{Figure 3}

Fgf10 mediates epithelial repair independent of Wnt ligands in part by activating/enhancing $\beta$-catenin signaling in the epithelium. $\beta$-gal staining $(\mathbf{A}-\mathbf{J})$ and $\beta$-gal staining with coimmunostaining for Scgb1a1 (K-O) or CGRP (P-T) on lungs 7 days after naphthalene treatment from TOPGAL (A, F, K, and P), dox-induced Rosa26-rtTA;Tet-Fgf10;TOPGAL (B, G, L, and Q), dox-induced Rosa26-rtTa;Tet-sFgfr2b;TOPGAL (C, H, M, and R), dox-induced Rosa26-rtTa;Tet-Dkk1;TOPGAL (D, I, N, and S), and dox-induced Rosa26-rtTa;Tet-Dkk1;Tet-Fgf10;TOPGAL mice (E, J, O, and T). Scale bars: 2,000 $\mu \mathrm{m}(\mathbf{A}-\mathbf{E}) ; 500 \mu \mathrm{m}(\mathbf{F}-\mathbf{J}) ; 50 \mu \mathrm{m}(\mathbf{K}-\mathbf{T})$.

different Wnt ligands in WT lungs 3 days after corn oil or naphthalene treatment by quantitative real-time PCR (qPCR) analysis using a Wnt signaling pathway qPCR array. Only Wnt7b expression levels were significantly changed, showing approximately 3 -fold upregulation 3 days after injury (Figure 1L and Supplemental Figure 2). Interestingly, a recent paper by Cohen et al. demonstrated that Wnt7b secreted by the airway epithelium plays an important role in PSMC development and in activation of the canonical Wnt pathway in PSMCs (18). The authors also demonstrated that the Wnt pathway becomes reactivated in the PSMCs in a mouse model for asthma, as well as in VSMCs in patients with pulmonary arterial hypertension (PAH) (18). Here we found that 3 days after naphthalene injury, surviving airway ciliated cells showed robust induction of Wnt7b expression (Figure 1, J and K), possibly activating surrounding PSMCs in a paracrine fashion. Interestingly, Fff10 expression was also induced in PSMCs after ozone- or bleomycin-mediated epithelial injury (Supplemental Figure 3, A-C).

Wnt-induced Fgf10 secreted by PSMCs is essential for epithelial repair after naphthalene injury. Next, we investigated the importance of Wnt-induced Fgf10, secreted by the PSMCs for epithelial regeneration after naphthalene injury. We generated 5 different mouse models, which we injured at 2 months of age with naphthalene. Rosa26-rtTA; Tet-sFgfr $2 b$ mice, which overexpress a dominant-negative soluble secreted $F g f r 2 b$ receptor $(s F g f r 2 b)$, were used to block
Fgf10 signaling by sequestering the Fgf10 ligand. Rosa26-rtTA;TetFgf10 mice were used to overexpress Fgf10. Rosa26-rtTA;Tet-Dkk1 mice were generated to overexpress Dkk1 and to suppress Fgf10 expression in the PSMCs. We generated Rosa26-rtTa;Tet-Dkk1; Tet-Fgf10 mice to conditionally overexpress both Dkk1 and Fgf10. Finally, we generated $M y b 11-c r e^{+} ; F g f 10^{f l / f l}$ mice to conditionally delete Fgf10 specifically in the SMCs (note that Myh11 is also known as smooth muscle myosin heavy chain [smMHC]).

Immunofluorescence and qPCR for $S c g b 1 a 1$ were used to assess and quantify the expansion of the Clara cells at different time points after injury. Inhibition of Fgf10 signaling by overexpression of the dominant-negative $F g f r 2 b$ receptor significantly impaired Clara cell regeneration after naphthalene injury (Figure 2, A, B, and I). On the other hand, overexpression of Fgf10 significantly accelerated airway epithelial regeneration (Figure 2, C and I). Mice overexpressing Dkk1 showed a significant impairment in regeneration 7 days after naphthalene injury (Figure 2I), and the majority did not survive up to 2 weeks after injury. The latter effect was not due to general overexpression of $D k k 1$, as noninjured doxycyclineinduced (dox-induced) Rosa26-rtTA;Tet-Dkk1 mice survived readily up to 6 months of age, after 4 months of continuous induction. However, the impaired regeneration was rescued by coexpressing Dkk1 and Fgf10: Rosa26-rtTA;Tet-Dkk1;Tet-Fgf10 mice showed accelerated epithelial repair, similar to that of mice overexpressing 

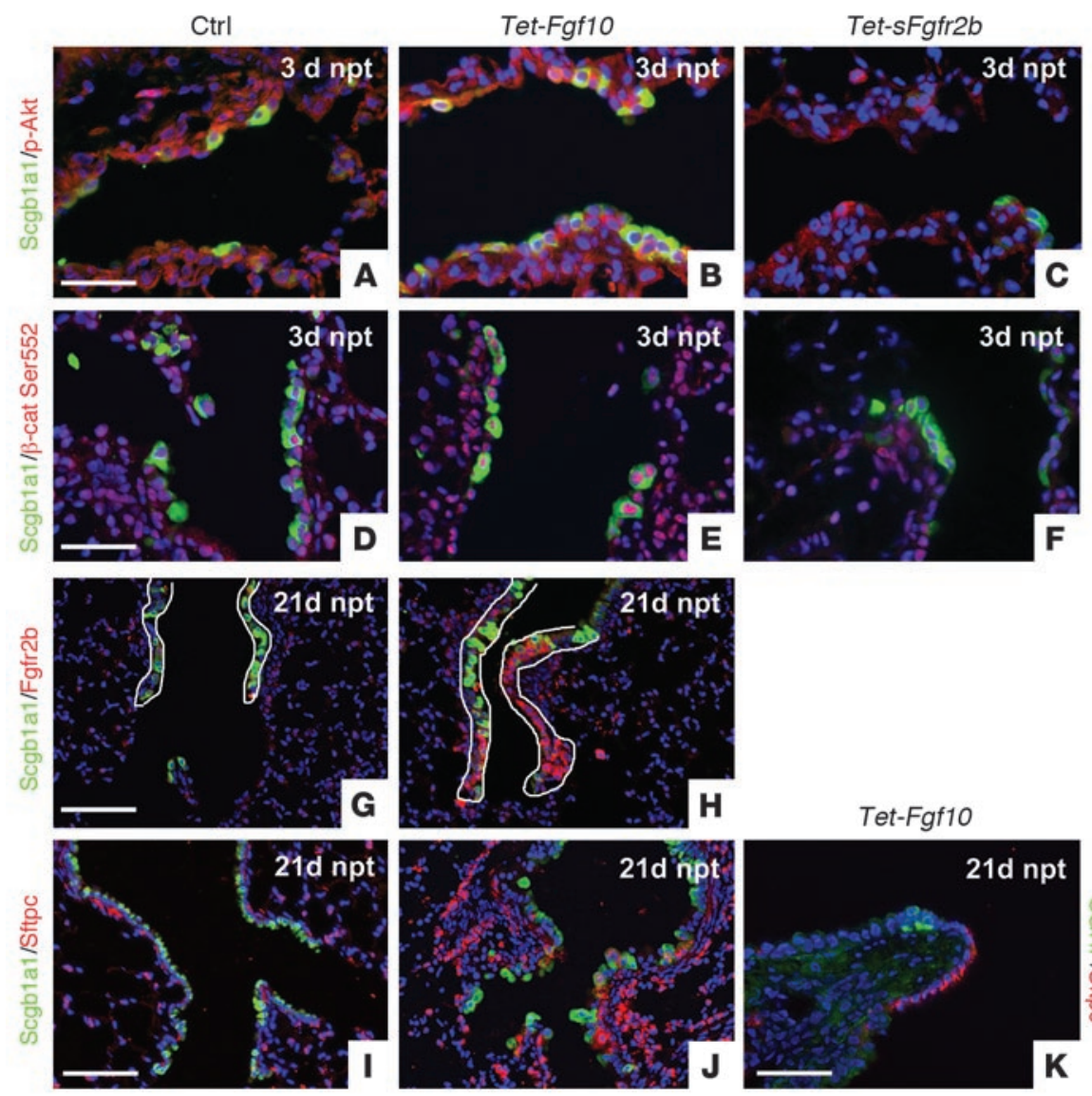

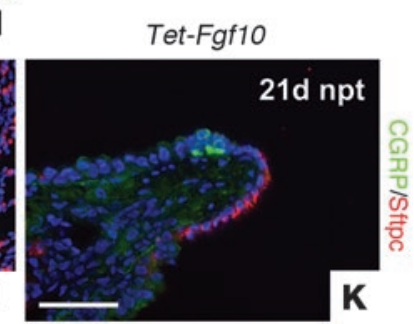

\section{Figure 4}

Fgf10 signaling induces Akt-mediated phosphorylation of $\beta$-catenin and maintenance/ amplification of variant Clara cells. (A-C) Immunostaining for $\mathrm{p}$-Akt and Scgb1a1 on lungs from control (A), Rosa26-rtTa;TetFgf10 (B), and Rosa26-rtTA;Tet-sFgfr2b (C) mice 3 days after naphthalene treatment. (D-F) Immunostaining for $p-\beta$-cateninSer552 and Scgb1a1 on lungs from control (D), Rosa26-rtTa;Tet-Fgf10 (E), and Rosa26rtTA;Tet-sFgfr2b (F) mice 3 days after naphthalene treatment. ( $\mathbf{G}$ and $\mathbf{H}$ ) Immunostaining for Scgb1a1 and Fgfr2b on lungs from control (G) and Rosa26-rtTa;Tet-Fgf10 (H) mice 21 days after naphthalene treatment. White outlines denote the epithelium at the BADJ. (I and J) Immunostaining for BASC markers Scgb1a1 and Sftpc on lungs from control (I) and Rosa26-rtTa;Tet-Fgf10 (J) mice 21 days after naphthalene treatment. (K) Immunostaining for CGRP and Sftpc on lungs from Rosa26-rtTa;Tet-Fgf10 mice 21 days after naphthalene treatment. Scale bars: $50 \mu \mathrm{m}$ (A-F and $\mathbf{K}) ; 100 \mu \mathrm{m}(\mathbf{G}-\mathbf{J})$. only Fgf10 (Figure 2, G-I). Together, these data suggest that the main mechanism by which $D k k 1$ overexpression impairs epithelial regeneration is inhibition of Wnt7b-induced Fgf10 expression in the PSMCs. Fgf10 affected the amplification of variant Clara cells at the BADJs as well as near the NEBs (Figure 2, A-K).

To confirm that PSMCs are indeed the source of Fgf10 important for regeneration, we performed naphthalene injury on $\mathrm{Myb11-Cre+}$; $\mathrm{Fg} f 10^{f / f l}$ mice. Clara cell regeneration was severely impaired in mice with SMC-specific deletion of Fgf10 (Figure 2, L-P). Moreover, qPCR analysis revealed a dramatic decrease in Fgf10 expression in lungs from Myb11-Cre; Fgf1 $0^{f / f l}$ versus control lungs 14 days after naphthalene injury (Supplemental Figure 1N). Because Myb11-Cre; $\mathrm{Fg} f 10^{f / f l}$ mice were very susceptible to injury, they were therefore injured suboptimally to allow for survival so that we could address the role of PSMC-derived Fgf10 in airway epithelial regeneration.

Fgf10 mediates airway epithelial repair independent of Wnt ligands, in part by directly activating/enhancing $\beta$-catenin signaling in the epithelium. Epithelial Wnt signaling has previously been shown to accelerate Clara cell regeneration after naphthalene injury and is known to amplify the variant Clara cells at the BADJs $(19,20)$. Our previous work has suggested that Fgf10 can activate $\beta$-catenin signaling directly in the epithelium $(8,13)$. To test whether Fgf10 directly activates $\beta$-catenin signaling during repair, we crossed the TOPGAL Wnt signaling reporter allele into the different mouse lines described above, performed naphthalene injury, and monitored epithelial $\beta$-catenin signaling 7 days after injury. At 7 days after naphthalene injury, lungs from control TOPGAL mice were partially regenerated and showed TOPGAL activity in the regenerat- ing Clara cells at the BADJs as well as near the NEBs (Figure 3, A, F, K, and P). Mice overexpressing Fgf10 showed a robust increase in Clara cell regeneration, as described above, and showed a strong increase in epithelial TOPGAL activity in the Clara cells at both the BADJs and the NEBs (Figure 3, B, G, L, and Q). Mice overexpressing $s F g$ fr $2 b$ or Dkk1 showed a profound decrease in Clara cell regeneration and had limited TOPGAL activity in the Clara cells at both BADJs and NEBs. This indicates that inhibition of Fgf10 signaling through either sequestering of the Fgf10 ligand (Figure 3, C, H, M, and R) or suppressing Fgf10 expression (Figure 3, D, I, N, and S) by overexpressing $s F g f r 2 b$ or $D k k 1$, respectively, inhibits not only Clara cell regeneration, but also epithelial $\beta$-catenin signaling.

Finally, we demonstrated that naphthalene-injured lungs from mice overexpressing both Dkk1 and Fgf10 showed a rescue in Clara cell regeneration as well as an increase in $\beta$-catenin signaling at the BADJs and NEBs (Figure 3, E, J, O, and T), which indicates that Fgf10 signaling can induce repair independent of Wnt ligands and activate the $\beta$-catenin signaling pathway directly.

Fgf10 signaling induces Akt-mediated phosphorylation of $\beta$-catenin and maintenance/amplification of variant Clara cells. The main mechanism through which Fgf10 is thought to activate $\beta$-catenin signaling is by activation of the PI3K-Akt pathway. Akt acts to inhibit GSK $3 \beta$, therefore preventing the degradation of $\beta$-catenin, as well as to phosphorylate $\beta$-catenin directly on Ser552 to drive it to the nucleus (21). To establish the mechanism by which Fgf10 activates $\beta$-catenin signaling, we performed immunostaining for phosphorylated Akt (p-Akt) and p- $\beta$-catenin-Ser552 on lung samples from control, Rosa26-rtTa;Tet-Fgf10, and Rosa26-rtTa-Tet-sFgr2b 

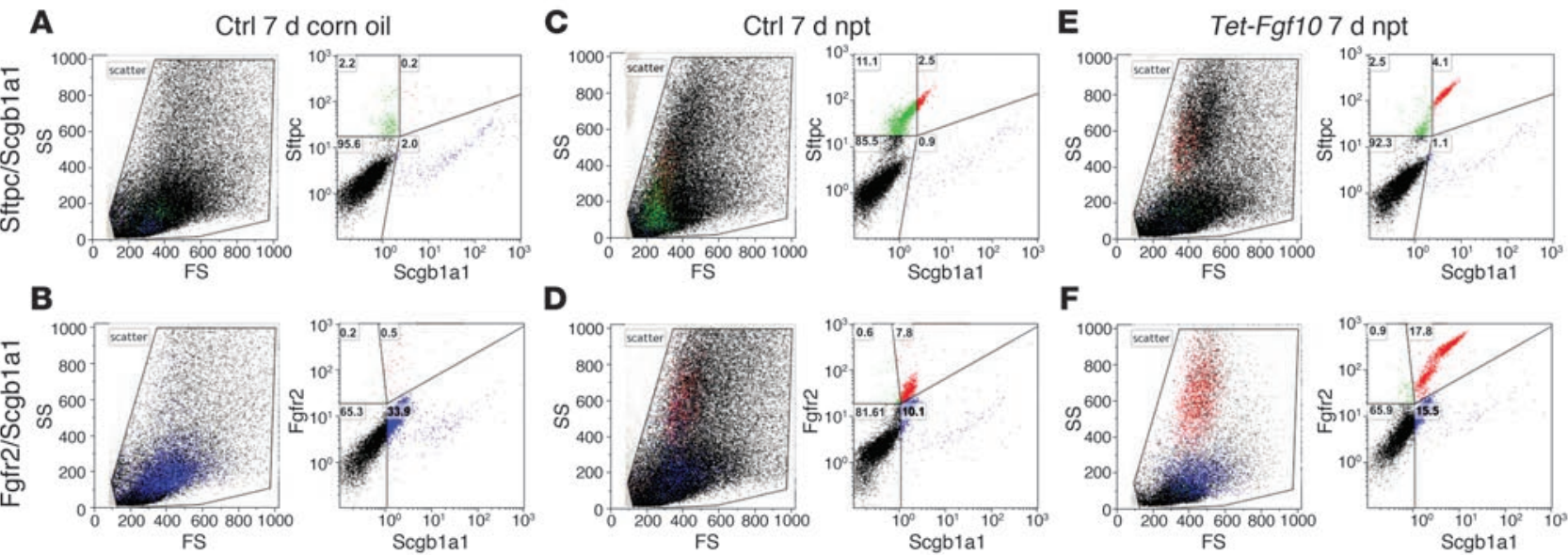

\section{Figure 5}

Flow cytometry analysis of airway epithelial stem cells. Flow cytometry analysis using Sftpc and Scgb1a1 (A, C, and E) versus Fgfr2 and Scgb1a1 (B, D, and F) antibodies on permeabilized epithelial cells from WT noninjured lungs ( $\mathbf{A}$ and $\mathbf{B})$, WT lungs 7 days after naphthalene injury (C and D), and Rosa26-rtTa;Tet-Fgf10 lungs 7 days after naphthalene injury (E and F). A 14-fold increase in double-positive airway epithelial cells was detected with both antibody combinations 7 days after naphthalene injury; a 22- to 33-fold increase was seen 7 days after injury in mice overexpressing Fgf10. Forward scatter/side scatter (FS/SS) plots indicated that the same pool of airway epithelial stem cells was isolated using either antibody combination.

mice 3 days after naphthalene injury. Modest phosphorylation of Akt and $\beta$-catenin was observed in control lungs (Figure 4, A and $\mathrm{D}$ ). This phosphorylation was increased in lungs overexpressing Fgflo (Figure 4, B and E) and dramatically decreased in lungs overexpressing sFgfr $2 b$ (Figure 4, C and F). We also observed phosphorylation of Akt and $\beta$-catenin-Ser552 in Clara cells at the BADJ after prolonged overexpression of Fgf10 in noninjured lungs (Supplemental Figure 4, I-L).

The putative stem cell population of variant Clara cells at the BADJ has been shown to be double positive for Sftpc and Scgb1a1 $(19,20,22)$; such cells have also been called bronchoalveolar stem cells (BASCs). Sftpc is a marker of not only alveolar epithelial type II cells, but also distal epithelial progenitor cells in the developing lung, where its expression is controlled by Fgf10. Therefore, reexpression of Sftpc in variant Clara cells could reflect an epithelial progenitor-like state. To analyze the effect of Fgf10 on the amplification and maintenance of variant Clara cells, we performed double immunostaining for Scgb1a1 and Sftpc on control and Fgf10-overexpressing lungs 21 days after naphthalene injury (Figure 4, I and J). Whereas an increase in variant Clara cells (Scgb1a1Sftpc double-positive cells) is normally seen shortly after naphthalene injury, these double-positive cells usually did not persist by 21 days after injury (Figure 4I). Interestingly, overexpression of Fgf10 not only amplified the number of BASCs after injury, but also maintained them as long as Fgf10 expression was induced (Figure 4J). These findings indicate that Fgf10, possibly (at least in part) by directly activating/enhancing epithelial $\beta$-catenin signaling, is important in generating variant Clara cells at the BADJs. Importantly, Fgf10 was also able to generate these Scgb1a1-Sftpc double-positive airway epithelial stem cells near the NEBs. Figure $4 \mathrm{~K}$ shows a cluster of Sftpc-positive Clara cells adjacent to a NEB in an Fgf10-overexpressing lung 21 days after naphthalene injury, which indicates that reexpression of Sftpc in variant Clara cells may be more universal in nature and possibly reflects partial dedifferentiation into a lung epithelial progenitor-like state. We also identified the Fgf10 receptor Fgfr2b as an extracellular marker for these Scgb1a1-Sftpc double-positive airway epithelial stem/progenitor cells (Figure 4, G and H, and see below). It is important to note that Fgfr2b is also a marker for distal embryonic lung epithelial progenitors and is downstream of Fgf10 as well as $\beta$-catenin signaling $(9,23)$. Amplification of Fgfr2b-expressing variant Clara cells at the BADJ was also observed after prolonged overexpression of Fgf10 in noninjured lungs and after ozone- and bleomycininduced lung injury (Supplemental Figure 4, A-D).

Flow cytometry analysis of Scgb1a1-Sftpc or Scgb1a1-Fgfr2 double-positive airway epithelial stem cells. To quantify the effect of naphthalene injury and Fgf10 expression on the generation of Scgb1a1-Sftpc or Scgb1a1-Fgfr2 double-positive cells, we performed flow cytometry analysis for these markers on permeabilized fixed single cell whole lung digests from noninjured control lungs as well as control and Fgf10-overexpressing lungs 7 days after naphthalene injury (Figure 5). In noninjured control lungs, a small fraction of the cells were Scgb1a1-Sftpc and Scgb1a1-Fgfr2 double-positive $(0.18 \%$ and $0.54 \%$, respectively; Figure 5, A and B). Injured control lungs showed an approximately 14-fold increase in Scgb1a1-Sftpc and Scgb1a1-Fgfr2 double-positive airway epithelial stem cells 7 days after injury relative to noninjured lungs (Figure 5, C and D). Finally, Fgf10-overexpressing lungs showed 22- and 33-fold increases, respectively, in Scgb1a1-Sftpc and Scgb1a1-Fgfr2 double-positive airway epithelial stem cells 7 days after injury (Figure 5, E and F). The forward scatter/side scatter plots in Figure 5 also illustrate that the same population of airway epithelial stem cells was isolated, by sorting for either the Scgb1a1 and Sftpc markers or the Scgb1a1 and Fgfr 2 markers. This identifies Fgfr $2 \mathrm{~b}$ as a marker for airway epithelial stem cells in the adult lung after naphthalene injury, a finding we believe to be novel.

Fgf10 signaling induces Fgfr $2 b$ expression, Notch activation, and subsequent Snail1 induction in activated variant Clara cells. Because of the importance of Fgf10 during lung development in maintaining the distal epithelial lung progenitors and preventing them from differ- 

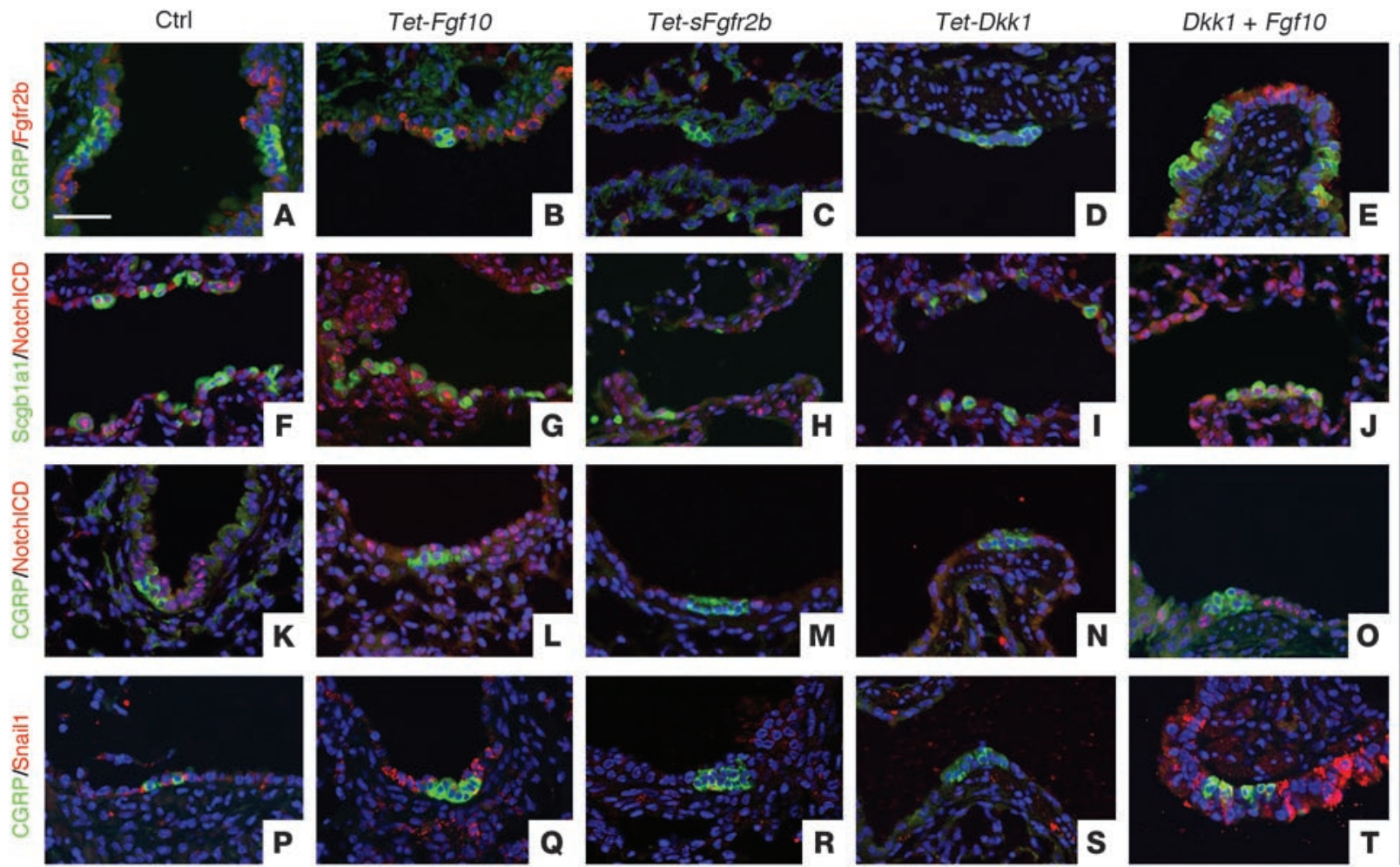

Figure 6

Fgf10 signaling induces Fgfr2b expression, Notch activation, and subsequent Snail induction in the activated variant Clara cells. Immunostaining for CGRP and Fgfr2b (A-E), Scgb1a1 and NotchICD (active notch) (F-J), CGRP and NotchICD (K-O), and CGRP and Snail1 (P-T) on lungs from control (A, F, K, and P), dox-induced Rosa26-rtTa;Tet-Fgf10 (B, G, L, and Q), dox-induced Rosa26-rtTA;Tet-sFgfr2b (C, H, M, and R), dox-induced Rosa26-rtTa;Tet-Dkk1 (D, I, N, and S), and dox-induced Rosa26-rtTa;Tet-Dkk1;Tet-Fgf10 (E, J, O, and T) mice 3 days after naphthalene treatment. Scale bar: $50 \mu \mathrm{m}(\mathbf{A}-\mathbf{T})$.

entiating into more proximal cell types, we next sought to unravel the mechanism by which Fgf10 generates airway epithelial stem cells. A recent paper by Mani and colleagues (24) established that in the mammary gland (another organ in which Fgf10 plays an important role; refs. 25, 26), the EMT generates mammary gland epithelial stem cells.

Our cell sorting data indicated that airway epithelial stem cells are positive for Fgfr $2 \mathrm{~b}$. Immunostaining 3 days after naphthalene injury demonstrated that regenerating Clara cells adjacent to NEBs were Fgfr2b positive in control and Fgf10-overexpressing lungs, but not in $s F g f r 2 b$ - or Dkk1-overexpressing lungs (Figure 6, A-D). Fgfr2b expression in Dkk1-overexpressing lungs was rescued by simultaneous overexpression of Fgf10 (Figure 6E). The most robust inducer of EMT is Snail1 (encoded by Snai1), which is tightly regulated on a transcriptional as well as a posttranslational level. Wnt, Fgf, and Notch pathways often act together to regulate Snail1 and induce an EMT program. Fgf and Notch have been previously shown to directly regulate Snail expression, whereas Fgf and Wnt, through inhibition of GSK3 $\beta$, act to stabilize the Snail 1 protein and protect it from degradation (reviewed in refs. 27-32).

Immunostaining for the cleaved Notch 1 intracellular domain (NotchICD) demonstrated that Notch signaling was activated in regenerating Clara cells/airway epithelial stem cells 3 days after injury at both BADJs and NEBs (Figure 6, F and K). Additionally, Notch activation was increased in lungs overexpressing Fgf10 and inhibited in lungs overexpressing $s F g$ fr $2 b$ or $D k k 1$, but was rescued upon overexpression of both Dkk1 and Fgf10 (Figure 6, F-O). Moreover, we found an induction in Snail1 expression in the regenerating Clara cells adjacent to NEBs and at BADJs in WT control lungs 3 days after naphthalene injury (Figure 6P and Supplemental Figure 1I). This induction in Snail1 was dramatically increased in lungs overexpressing Fgf10, inhibited in lungs overexpressing $s F g f r 2 b$ or $D k k 1$, and rescued upon overexpression of both $D k k 1$ and Fgf10 (Figure 6, P-T, and Supplemental Figure 1, K and L).

Variant Clara cells undergo a transient EMT in response to Fgf10-induced Notch activation and subsequent Snail1 induction. Finally, we sought to determine whether regenerating (naphthalene-resistant) Clara cells are indeed undergoing an EMT to generate airway epithelial stem cells. We crossed Myb11-Cre mice, which express the Cre recombinase under control of the smMHC promoter, with Rosa26R-eYFP or Rosa26R-LacZ reporter mice. During normal homeostasis, Myb11-Cre; Rosa26R mice only labeled airway and VSMCs in the lung, never lung epithelial cells. However, we observed labeled epithelial cells around 3 days after naphthalene injury (Figure 7A), which indicates that Clara cells undergo a transient EMT to generate airway epithelial stem cells or adopt epithelial stem cell properties. The labeled clusters expanded to eventually give rise to regenerated Clara cells (Figure 7, B and C). Rosa26R-labeled Clara cells were detected near both NEBs and BADJs (Figure 7, D and E). We further checked for expression of other markers consistent with EMT; we found loss of E-cadherin and increased smMHC and vimentin in Clara cells 3 days after naphthalene injury (Supplemental Figure 4, M-O). 

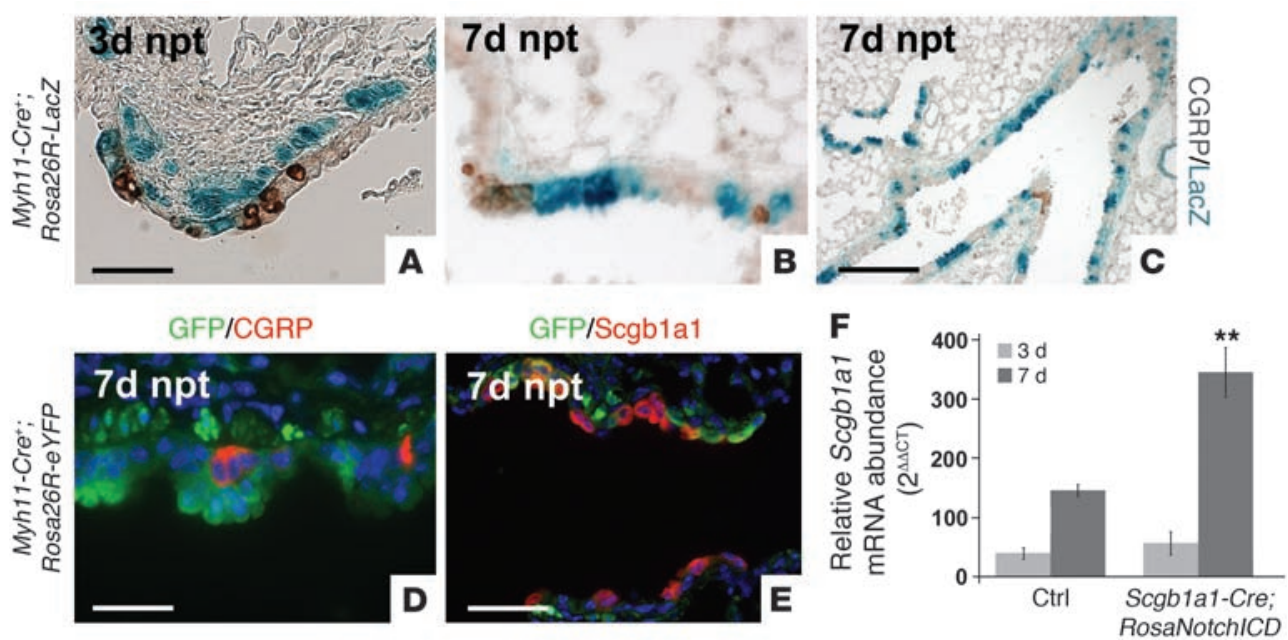

Ctrl
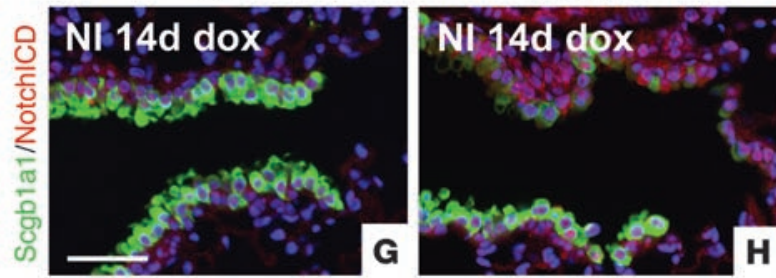

Scgb1a1-Cre;RosaNotchICD
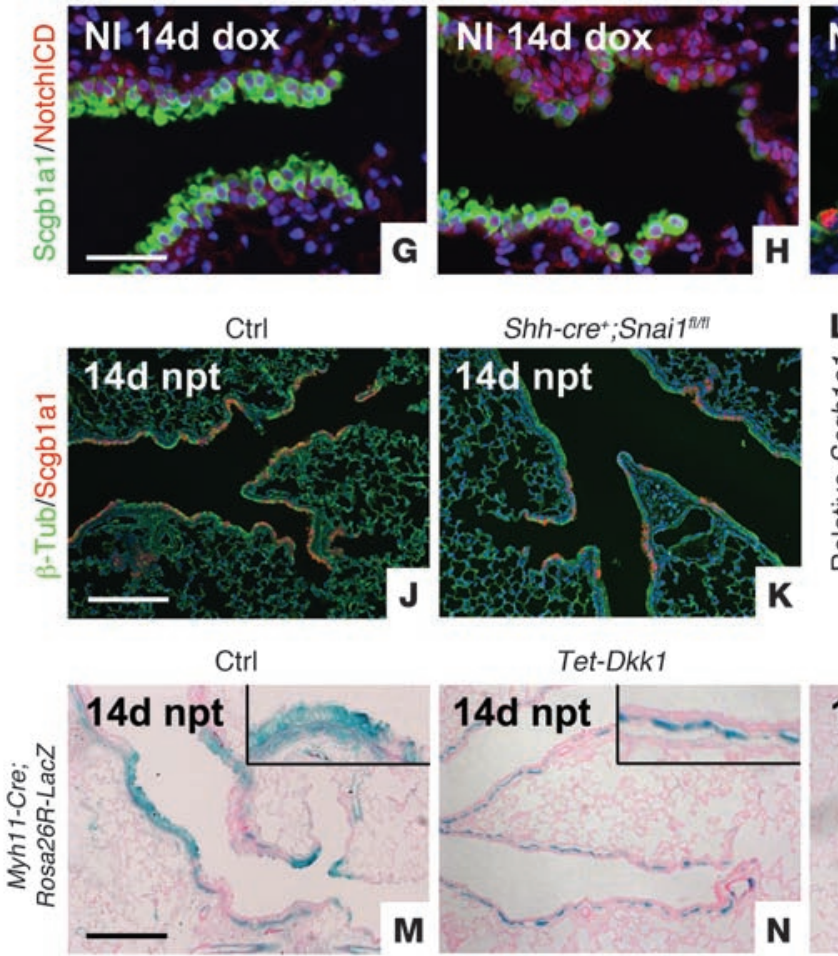
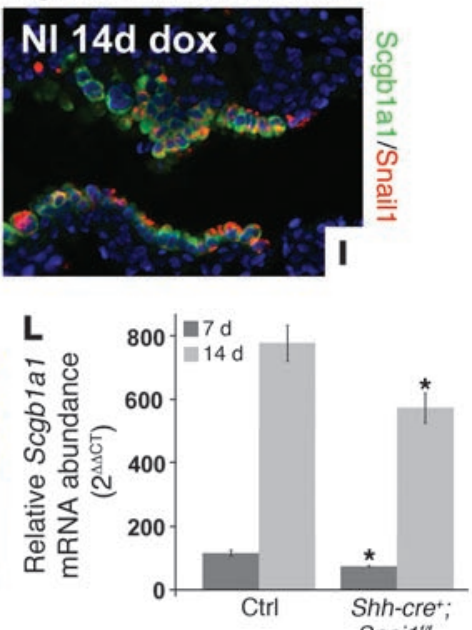

Tet-sFgfr2b

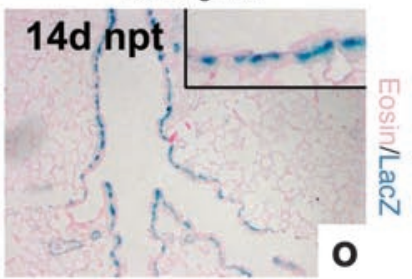

Figure 7

Variant Clara cells undergo transient EMT in response to Fgf10-induced Notch activation and subsequent Snail induction. (A-C) Lineage tracing of activated variant Clara cells that underwent transient EMT in Myh11-Cre;Rosa26-LacZ mice 3 (A) and 7 (B and C) days after naphthalene injury. (C) Lower-magnification view of $\mathbf{B}$. ( $\mathbf{D}$ and $\mathbf{E}$ ) Lineage tracing of activated variant Clara cells that underwent transient EMT in Myh11-Cre;Rosa26-eYFP mice 7 days after naphthalene injury, shown by immunostaining for GFP and CGRP (D) or GFP and Scgb1a1 (E). (F) qPCR analysis of relative Scgb1a1 mRNA abundance in lungs from 2-month-old control and Scgb1a1-rtTa;Tet-o-Cre;Rosa26-Notch/CD mice 3 and 7 days after naphthalene treatment. (G and $\mathbf{H})$ Immunostaining for NotchICD and Scgb1a1 on noninjured (NI) lungs from control (G) and Rosa26-rtTa;Tet-Fgf10 mice (H) 14 days after dox treatment. (I) Immunostaining for Snail1 and Scgb1a1 on noninjured lungs from Scgb1a1-rtTa;Tet-o-Cre;Rosa26-NotchlCD+/- mice 14 days after dox treatment. ( $\mathbf{J}$ and $\mathbf{K}$ ) Immunostaining for Scgb1a1 and $\beta$-tubulin on lungs from control (J) and Shh-Cre ${ }^{+}$;Snai1 ${ }^{f l / f l}(\mathbf{K})$ mice 14 days after naphthalene treatment. (L) qPCR analysis of relative Scgb1a1 mRNA abundance in lungs from 2-month-old control and Shh-Cre+;Snai1 ${ }^{f / f l}$ mice 7 and 14 days after naphthalene treatment. (M-O) Lineage tracing of activated variant Clara cells that underwent transient EMT in Myh11-Cre;Rosa26-LacZ (M), Myh11-Cre;Rosa26-LacZ;Rosa26-rtTa;Tet-Dkk1 (N), and Myh11-Cre;Rosa26-LacZ;Rosa26-rtTA; Tet-sFgfr2b (0) mice 14 days after naphthalene injury. Insets are enlarged $x 4$. ${ }^{* *} P<0.01,{ }^{*} P<0.05$ vs. control. $n \geq 3$. Scale bars: $50 \mu \mathrm{m}$ (A, B, E, and G-I); $200 \mu \mathrm{m}$ (C, J, K, and M-O); $31.7 \mu \mathrm{m}$ (D).

Additionally, we performed bleomycin injury on $\mathrm{Myb11-Cre}{ }^{+}$; Rosa26R-LacZ mice, which demonstrated that bleomycin injury also induced a transient EMT in a nonfibrotic area of airway epithelium 21 days after bleomycin injury (Supplemental Figure 4P).

To determine whether Fgf10, via induction of Notch signaling, induces Snail expression in Clara cells and therefore induces this transient EMT, we overexpressed Fgf10 in 2-monthold noninjured lungs for 14 and 21 days and found that prolonged Fgf10 exposure induced Snail expression and Notch activation in Clara cells at the BADJ in the absence of injury (Figure 7, G and H, Supplemental Figure $1, \mathrm{~K}-\mathrm{M}$, and Supplemental Figure 4, E and F). Furthermore, Snail1 was induced in Clara cells at BADJs after ozone or bleomycin injury (Supplemental Figure $4, \mathrm{G}$ and $\mathrm{H}$ ). To determine the role of Notch signaling directly in the generation of lung airway epithelial stem cells, we generated Scgb1a1-rtTa; Tet-O-Cre; Rosa26-NotchICD mice, allowing us to inducibly overexpress the NotchICD activator domain in Clara cells. Interestingly, noninjured lungs from Scgb1a1-rtTa; Tet-O-Cre;Rosa26-NotchICD mice looked normal, but showed robust Snail1 expression in Clara cells (Figure 7I). We then tested whether airway epithelial regeneration after naphthalene injury is increased in Scgb1a1-rtTa; Tet-O-Cre;Rosa26-NotchICD mice induced 3 days prior to injury. We found that activation of Notch signaling in Clara cells indeed accelerated regeneration of the airway epithelium after naphthalene injury (Figure 7F). To investigate the importance of Snail1 in airway epithelium regeneration after naphthalene injury, we generated Shb-Cre ${ }^{+}$;Snai1 ${ }^{f l / f l}$ mice, in which Snai1 is conditionally deleted from all lung epithelial cells, and found that Snail1 in Clara cells undergoing repair was important for the proper restoration and function of the 
A Developing lung

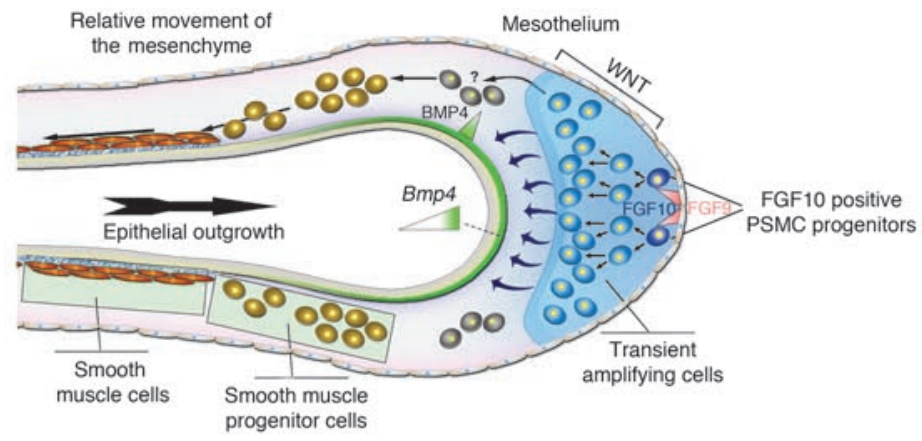

B Adult lung
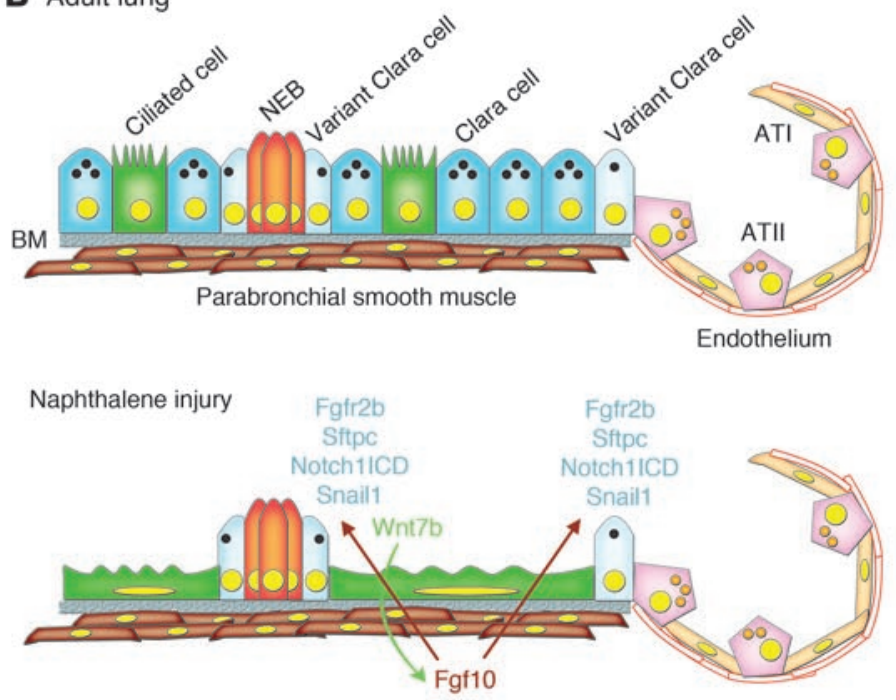

In repairing state

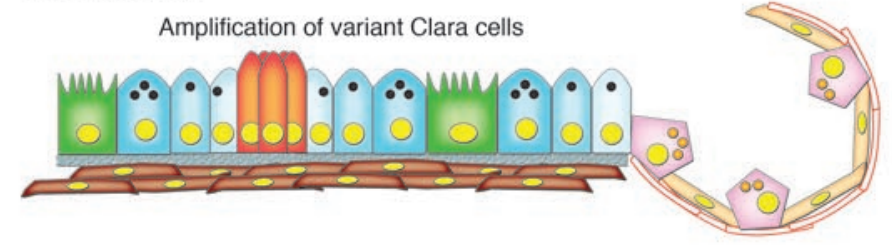

\section{Figure 8}

Model of PSMC progenitors during development and recapitulation of a progenitor state by mature PSMCs after naphthalene injury. (A) During embryonic lung development, PSMC progenitors are identified as Fgf10-expressing cells in the distal mesenchyme (10). The amplification of these PSMC progenitors as well as their Fgf10 expression is regulated by mesenchymal $\beta$-catenin signaling (9). Fgf10 signaling is critical during lung development in the maintenance of lung epithelial progenitors $(5,8,13)$. (B) In the adult lung, Fgf10 expression in mature PSMCs is silenced. Upon naphthalene administration, Clara cells are destroyed - except for variant Clara cells - at both NEBs and BADJs. Ciliated cells spread out to cover the basement membrane (BM) and induce Wnt7b expression. Wnt7b then acts on the PSMCs to reactivate Fgf10 expression, which then acts on the variant Clara cells to activate Notch signaling and induce expression of Fgfr2b, Sftpc, and Snail1, thereby initiating the repair process through a transient EMT. ATI, alveolar type I cell; ATII, alveolar type II cell; NEB, neuroendocrine body.

we found that this Wnt/Fgf10 embryonic signaling cascade was reactivated in mature PSMCs after naphthalene-induced Clara cell epithelial injury.

In the adult lung after epithelial injury, surviving airway ciliated cells induced Wnt7b expression, which then acted on surrounding PSMCs to induce Fgf10 expression (Figure $8 \mathrm{~B})$. In response to $\mathrm{Wnt} 7 \mathrm{~b}$, mature PSMCs recapitulated an embryonic progenitor-like state, began to proliferate, and secreted Fgf10 to stimulate epithelial repair through a transient EMT by inducing Snai1 expression (Figure 8B). Our data suggest that this transient EMT allows the surviving variant Clara cells to adopt an epithelial stem cell/progenitor state characterized by Sftpc, Fgfr2b, and Snail1 expression.

Diseases such as cancer, and proliferative lung diseases such as fibrosis, may adopt and exploit the mechanisms by which the body normally renews itself. During the processes of tumor metastasis and fibrosis, which are often enabled by EMTs (30-32), cells demonstrate a capability for self-renewal similar to that exhibited by stem cells in order to expand and/or colonize different tissues. This raises the possibility that the EMT process may impart a self-renewal capability to epithelial cells. Recently, Mani et al. (24) reported that an EMT induction in immortalized human mammary epithelial cells results in the acquisition of mesenchymal traits and the expression of stem cell markers. In addition, they showed that those cells have an increased ability to form mammospheres, a property associated with mammary epithelial stem cells. Moreover, stem-like cells isolated from either mouse or human mammary glands or mammary carcinomas express EMT markers. These findings illustrate a direct link between the EMT and the gain of epithelial stem cell properties. Together, these diverse lines of evidence suggest a possible link between less-differentiated stem cells and the mesenchymal-appearing cells generated by EMTs. Our data not only indicate that after lung epithelial injury, Fgf10 signaling is important in maintaining an epithelial progenitor state (as during lung development), but suggest that Fgf10 may also impart a stem cell state with self-renewal capability by initiating a transient EMT. The adoption of a stem cell state and the transient EMT seems to be regulated, at least in part, by Snail1. However, considering the fairly modest decrease in regeneration in $\mathrm{Shb}_{\mathrm{Cre}}^{+}$;Snai1 ${ }^{\mathrm{fl} / \mathrm{fl}}$ mice, it is likely that some alternate or redundant proteins like Snail2 (also known as Slug) may play a role as well. 
We demonstrated that induction of Fgf10 expression by the PSMCs and subsequent activation of Clara cells at the BADJs, based on Fgfr2b and Snail1 expression (Supplemental Figure 3 and Supplemental Figure 4, C, D, G, and H), was part of a repair mechanism induced by several types of airway epithelial injury. Our findings pinpoint some of the similar signaling pathways that occur during lung development and are invoked in the injury response in the adult lung to properly regenerate damaged and lost cell lineages. However, a fine balance in the level of reactivation of these pathways needs to be found in order for proper regeneration to occur; too much or too little can lead to abnormal repair and remodeling (reviewed in ref. 33). Our data suggest that under conditions of chronic injury, the lung may invoke these same signaling pathways; however, sustained stimulation of these pathways may eventually lead to full EMT, resulting in epithelial stem cell depletion and fibrosis. Interestingly, the Wnt pathway has previously been shown to be induced in PSMCs in a mouse model for asthma (18), whereas signaling of epithelial Fgf10 as well as Notch have been shown to be implicated in mucous/goblet cell hyperplasia $(6,34)$. Unlike human lungs, mouse lungs have very few goblet cells in the upper airways, and recent evidence indicates that Clara cells transdifferentiate into goblet cells $(34,35)$ in response to allergen. We demonstrated that PSMC-secreted Fgf10 induced Clara cell-to-goblet cell transdifferentiation in the repairing upper airway after naphthalene injury (Supplemental Figure 5), possibly by activating the Notch pathway. Therefore, we hypothesize that overactivation of this Wnt-Fgf10 epithelial-mesenchymal cross-talk may be involved in airway remodeling in response to chronic injury in asthma patients. Future studies will be required to address this.

\section{Methods}

Mouse strains. Tet-Dkk1 mice were generated by knocking in a Tet-Dkk1 cassette in the Hprt locus through homologous recombination. CMV-Cre mice [c-Tg(CMV-cre) $1 \mathrm{Cgn} / \mathrm{J}$; Jackson Laboratories] were crossed with Rosa26rtTAflox mice (36) to generate Rosa26-rtTA mice expressing rtTA from the Rosa26 promoter in every cell of the body. Rosa26-rtTA mice were crossed with Tet-sFgfr2b (37), Tet-Fgf10 (38), and Tet-Dkk1 mice to generate doubletransgenic mice. These mice were on the mixed genetic background and allowed inducible expression of $s F g f r 2 b, F g f 10$, and $D k k 1$, respectively, by feeding mice food containing dox (rodent diet with $625 \mathrm{mg} / \mathrm{kg}$ dox; Harlan Teklad TD.09761). TOPGAL mice were a gift from E. Fuchs (Rockefeller University, New York, New York, USA; ref. 14). Fgf1 $10^{\text {fl/l }}$ mice were a gift from S. Mansour (University of Utah, Salt Lake City, Utah, USA; ref. 39). Myb11Cre [Tg(Myb11-cre,-EGFP)2Mik/J], Rosa26R-LacZ [Gt(Rosa)26Sortm1Sor], Rosa26R-eYPF [Gt(Rosa)26Sortm1(eYFP)Cos], Rosa26-NotchICD [Tg(CAG-Bgeo, -NOTCH1,-EGFP)1Lbe/J], Scgb1a1-rtTA [Tg(Scgb1a1-rtTA)1Jaw/J], Tet-O-Cre

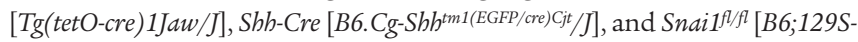
Snai $1^{\text {tm } 2 \text { Grid } / J]}$ mice were obtained from Jackson Laboratories. Adult mice were 8 weeks old at time of naphthalene administration. Animals were maintained in a pathogen-free environment.

$\beta$-gal staining. Tissues containing Rosa26R, TOPGAL, or Fgf $10^{\text {LacZ }}$ alleles were dissected, and $\beta$-gal staining was performed at different stages after naphthalene injury $(3,7,14$, and 21 days). Lungs were dissected and fixed in $4 \%$ PFA in PBS at room temperature for 5 minutes, rinsed in PBS, injected with freshly prepared $\mathrm{X}$-gal solution, transferred into a vial of $\mathrm{X}$-gal solution, and stained at $37^{\circ} \mathrm{C}$ overnight. After rinsing with $\mathrm{PBS}$, lungs were postfixed in 4\% PFA in PBS at room temperature overnight. For microtome sections, after 4\% PFA fixation, lungs were washed in PBS, dehydrated, and paraffin embedded. For clearing, after 4\% PFA fixation, lungs were washed in PBS, dehydrated, and cleared with BABB (1:2 benzyl alcohol/benzyl benzoate) as follows: tissue transferred to 1:2 BABB/ ethanol for 20 minutes, $2: 1 \mathrm{BABB} /$ ethanol for 20 minutes, and $100 \%$ BABB for 20 minutes.

Immunohistochemistry and fluorescence. All staining was done on paraffin sections of formalin-fixed lungs. Immunohistochemistry was performed with the Histostain-Plus Kit (Invitrogen). Immunohistochemistry and fluorescent staining was performed with the following primary antibodies: mouse anti- $\beta$-tubulin (3F3-G2; Seven Hills Bioreagents), goat anti-Wnt7b (R\&D Systems), goat anti-Scgb1a1 (T-18; Santa Cruz Biotechnology Inc.), rabbit anti-Scgb1a1 (Seven Hills Bioreagents), rabbit anti-CGRP (Sigma-Aldrich), chicken anti-CGRP (Neuromics), rabbit anti-Fgfr2 (Bek) (C-17; Santa Cruz Biotechnology Inc.), rabbit anti-activated Notch1 (Abcam), goat anti-Snail1 (E-18; Santa Cruz Biotechnology, Inc.), mouse anti- $\alpha$-SMA cy 3 conjugate and unconjugated (14A; SigmaAldrich), chicken anti-GFP (Aves Labs Inc.), rabbit anti-Sftpc (Seven Hills Bioreagents), rabbit anti- $\beta$-catenin-Ser552 (Cell Signaling Technology), mouse anti-Vimentin (Sigma-Aldrich), rabbit anti- $\beta$-gal (Rockland Immunochemicals Inc.), mouse anti-E-cadherin (BD Transduction Laboratories), mouse anti-smMHC (1C10; Santa Cruz Biotechnology Inc.), and rabbit anti-p-Akt Ser473 (D9E; Cell Signaling Technology). All fluorescent staining was performed with secondary antibodies from Jackson Immunoresearch (except the Cy3-conjugated $\alpha$-SMA) and mounted using Vectashield with DAPI (Vector Labs).

qPCR. RNA was isolated from lung accessory lobes using RNALater (Ambion) and Total RNA Kit I (Omega Biotek) according to the manufacturer's instructions. RNA concentration was determined by spectrophotometry. cDNA was generated using SuperScript III First-Strand Synthesis System (Invitrogen) according to the manufacturer's instructions. Comparative real-time PCR was performed for $\beta$-glucuronidase (Mm00446953_m1), Scgb1a1 (Mm00442046_m1), Fgf10 (Mm01297079_ m1), Snai1 (Mm00441533_g1), Wnt3a (Mm00437337_m1), and Wnt7b (Mm00437358_m1) Taqman Gene Expression Assays (Applied Biosystems) using a StepOne Plus system (Applied Biosystems). $\beta$-glucuronidase was used as a reference control to normalize equal loading of template cDNA.

qPCR array. RNA was isolated from lung accessory lobes using RNALater (Ambion) and RNeasy Mini Kit (Qiagen) according to the manufacturer's instructions. cDNA was synthesized as described above. Quantitative PCR was performed on a mouse Wnt signaling pathway array via the RT ${ }^{2}$ Profiler PCR Array (SABiosciences) on a StepOne Plus system (Applied Biosystems).

Naphthalene treatment. Naphthalene (Sigma-Aldrich) was dissolved in corn oil at $30 \mathrm{mg} / \mathrm{ml}$ and administered intraperitoneally at 8 weeks of age, with doses adjusted according to strain to achieve a $95 \%$ decrease in the abundance of Scgb1a1 mRNA in total lung RNA of WT mice at 3 days after injection. Control mice for regeneration studies were WT littermates.

Flow cytometry analysis. Cells were prepared from noninjured control mice and from control and Rosa26-rtTA;Tet-Fgf10 mice 7 days after naphthalene injury using Collagenase type 2 (Worthington) and Red Blood Cell Lysis Buffer (eBioscience). Live/dead staining was performed with LIVE/DEAD Fixable Violet Dead Cell Stain Kit (Invitrogen). Cells were fixed and permeabilized with Perm/Wash Buffer and Cytofix/Cytoperm (BD). Cells were then stained with antibodies against Sftpc (Seven Hills Bioreagents), Fgfr2 (Bek) (Santa Cruz Biotechnology Inc.), or Scgb1a1 (Santa Cruz Biotechnology Inc.) and labeled with Zenon Alexa Fluor Labeling Kits (Invitrogen). Sorting was performed on a CYAN (Dako) with Kaluza software.

Proliferation. Mice were given intraperitoneal injections of $10 \mu \mathrm{BrdU}$ (GE Healthcare) per gram body weight 4 hours before sacrifice. Lungs were fixed in $4 \%$ paraformaldehyde, dehydrated, and paraffin embedded. Sec- 
tions were treated with monoclonal anti-BrdU (clone BU-1; GE Healthcare) according to the manufacturer's instructions. FITC-labeled anti-mouse secondary antibodies were used (Jackson Immunoresearch). All slides were mounted using Vectashield with DAPI.

In situ bybridization. In situ hybridization on $10-\mu \mathrm{m}$ paraffin sections of formalin-fixed lungs was performed as previously described $(9,13)$. A 584-bp Fgf10 mouse cDNA (5) and a full-length Snai1 mouse cDNA (subcloned by PCR using forward and reverse Snail primers CTAGGTCGCTCTGGCCAAC and GAGGATGGGGAGGTAGCAG, respectively) were used as templates for the synthesis of digoxigenin-labeled antisense riboprobes.

Statistics. For BrdU labeling, qPCR, and fluorescence intensity analysis, each experiment was repeated with the samples obtained from at least 3 different lungs preparations. All results are expressed as mean \pm SEM. The significance of differences between 2 sample means was determined by a 2 -tailed Student's $t$ test. $P$ values less than 0.05 were considered statistically significant.

Study approval. All experiments were approved by the National Jewish Health institutional animal care and use committee.

\section{Acknowledgments}

These studies were supported by funding from the NIH to S.P. De Langhe (grants HL092967 and HL074832) and to S. Bellusci (grant HL074832). S.P. De Langhe also acknowledges National Jewish Health institutional start up funds. S. Bellusci also acknowledges funding from the Excellence Cluster in Cardio Pulmonary System and the DFG. C. Tiozzo acknowledges funding from ALA and CIRM. The authors thank L. Niswander for helpful suggestions and critical reading of the manuscript; S. Mansour for providing the Fgf1 $10^{f / f l}$ mice, and the CU cancer center flow cytometry core and Christine Childs.

Received for publication March 18, 2011, and accepted in revised form August 24, 2011.

Address correspondence to: Stijn De Langhe, Department of Pediatrics, National Jewish Health, Goodman K1003A, 1400 Jackson Street, Denver Colorado 80206, USA. Phone: 303.398.1763; Fax: 303.398.1225; E-mail: delanghes@njhealth.org.
1. Giangreco A, Reynolds SD, Stripp BR. Terminal bronchioles harbor a unique airway stem cell population that localizes to the bronchoalveolar duct junction. Am J Pathol. 2002;161(1):173-182.

2. Reynolds SD, Giangreco A, Power JH, Stripp BR. Neuroepithelial bodies of pulmonary airways serve as a reservoir of progenitor cells capable of epithelial regeneration. Am J Pathol. 2000;156(1):269-278.

3. Chen H, Matsumoto K, Stripp BR. Bronchiolar progenitor cells. Proc Am Thorac Soc. 2009;6(7):602-606.

4. Rawlins EL, Hogan BL. Epithelial stem cells of the lung: privileged few or opportunities for many? Development. 2006;133(13):2455-2465.

5. Bellusci S, Grindley J, Emoto H, Itoh N, Hogan BL. Fibroblast growth factor 10 (FGF10) and branching morphogenesis in the embryonic mouse lung. Development. 1997;124(23):4867.

6. Nyeng P, Norgaard GA, Kobberup S, Jensen J. FGF10 maintains distal lung bud epithelium and excessive signaling leads to progenitor state arrest, distalization, and goblet cell metaplasia. BMC Dev Biol. 2008;8:2.

7. Park WY, Miranda B, Lebeche D, Hashimoto G, Cardoso WV. FGF-10 is a chemotactic factor for distal epithelial buds during lung development. Dev Biol. 1998;201(2):125-134.

8. Ramasamy SK, et al. Fgf10 dosage is critical for the amplification of epithelial cell progenitors and for the formation of multiple mesenchymal lineages during lung development. Dev Biol. 2007;307(2):237-247.

9. De Langhe SP, et al. Formation and Differentiation of Multiple Mesenchymal Lineages during Lung Development Is Regulated by beta-catenin Signaling. PLoS One. 2008;3(1):e1516.

10. Mailleux AA, et al. Fgf10 expression identifies parabronchial smooth muscle cell progenitors and is required for their entry into the smooth muscle cell lineage. Development. 2005;132(9):2157.

11. Goss AM, et al. Wnt2 signaling is necessary and sufficient to activate the airway smooth muscle program in the lung by regulating myocardin/Mrtf-B and Fgf10 expression. Dev Biol. 2011;356(2):541-552.

12. De Langhe SP, et al. Dickkopf-1 (DKK1) reveals that fibronectin is a major target of Wnt signaling in branching morphogenesis of the mouse embryonic lung. Dev Biol. 2005;277(2):316-331.

13. De Langhe SP, Carraro G, Warburton D, Hajihosseini
MK, Bellusci S. Levels of mesenchymal FGFR2 signaling modulate smooth muscle progenitor cell commitment in the lung. Dev Biol. 2006;299(1):52-62.

14. DasGupta R, Fuchs E. Multiple roles for activated LEF/TCF transcription complexes during hair follicle development and differentiation. Development. 1999;126(20):4557-4568.

15. Kelly RG, Brown NA, Buckingham ME. The arterial pole of the mouse heart forms from Fgf10expressing cells in pharyngeal mesoderm. Dev Cell. 2001;1(3):435-440.

16. Mao B, et al. Kremen proteins are Dickkopf receptors that regulate $\mathrm{Wnt} /$ beta-catenin signalling. Nature. 2002;417(6889):664-667.

17. Mao B, et al. LDL-receptor-related protein 6 is a receptor for Dickkopf proteins. Nature. 2001; 411(6835):321-325.

18. Cohen ED, Ihida-Stansbury K, Lu MM, Panettieri RA, Jones PL, Morrisey EE. Wnt signaling regulates smooth muscle precursor development in the mouse lung via a tenascin C/PDGFR pathway. JClin Invest. 2009;119(9):2538-2549.

19. Reynolds SD, et al. Conditional stabilization of beta-catenin expands the pool of lung stem cells. Stem Cells. 2008;26(5):1337-1346.

20. Zhang Y, et al. A Gata6-Wnt pathway required for epithelial stem cell development and airway regeneration. Nat Genet. 2008;40(7):862-870.

21. He XC, et al. PTEN-deficient intestinal stem cells initiate intestinal polyposis. Nat Genet. 2007; 39(2):189-198.

22. Kim CF, et al. Identification of bronchioalveolar stem cells in normal lung and lung cancer. Cell. 2005; 121(6):823-835.

23. Shu W, et al. Wnt/beta-catenin signaling acts upstream of $\mathrm{N}$-myc, BMP4, and FGF signaling to regulate proximal-distal patterning in the lung. Dev Biol. 2005;283(1):226-239.

24. Mani SA, et al. The epithelial-mesenchymal transition generates cells with properties of stem cells. Cell. 2008;133(4):704-715.

25. Mailleux AA, et al. Role of FGF10/FGFR2b signaling during mammary gland development in the mouse embryo. Development. 2002;129(1):53-60.

26. Parsa $S$, et al. Terminal end bud maintenance in mammary gland is dependent upon FGFR2b sig- naling. Dev Biol. 2008;317(1):121-131.

27. Acloque H, Adams MS, Fishwick K, Bronner-Fraser M, Nieto MA. Epithelial-mesenchymal transitions: the importance of changing cell state in development and disease. J Clin Invest. 2009;119(6):1438-1449.

28. Barrallo-Gimeno A, Nieto MA. The Snail genes as inducers of cell movement and survival: implications in development and cancer. Development. 2005; 132(14):3151-3161.

29. Thiery JP. Epithelial-mesenchymal transitions in tumour progression. Nat Rev Cancer. 2002; 2(6):442-454.

30. Thiery JP. Epithelial-mesenchymal transitions in development and pathologies. Curr Opin Cell Biol. 2003;15(6):740-746.

31. Thiery JP, Acloque H, Huang RY, Nieto MA. Epithelial-mesenchymal transitions in development and disease. Cell. 2009;139(5):871-890.

32. Thiery JP, Sleeman JP. Complex networks orchestrate epithelial-mesenchymal transitions. Nat Rev Mol Cell Biol. 2006;7(2):131-142.

33. Beers MF, Morrisey EE. The three R's of lung health and disease: repair, remodeling, and regeneration. J Clin Invest. 2011;121(6):2065-2073.

34. Guseh JS, et al. Notch signaling promotes airway mucous metaplasia and inhibits alveolar development. Development. 2009;136(10):1751-1759.

35. Tompkins DH, et al. Sox 2 is required for maintenance and differentiation of bronchiolar Clara, ciliated, and goblet cells. PLoS One. 2009;4(12):e8248.

36. Belteki G, et al. Conditional and inducible transgene expression in mice through the combinatorial use of Cre-mediated recombination and tetracycline induction. Nucleic Acids Res. 2005;33(5):e51.

37. Hokuto I, Perl AK, Whitsett JA. Prenatal, but not postnatal, inhibition of fibroblast growth factor receptor signaling causes emphysema. J Biol Chem. 2003;278(1):415-421.

38. Clark JC, et al. FGF-10 disrupts lung morphogenesis and causes pulmonary adenomas in vivo. Am J Physiol Lung Cell Mol Physiol. 2001;280(4):L705-L715.

39. Urness LD, Paxton CN, Wang X, Schoenwolf GC, Mansour SL. FGF signaling regulates otic placode induction and refinement by controlling both ectodermal target genes and hindbrain Wnt8a. Dev Biol. 2010;340(2):595-604. 